venous drainage due to previous surgery. Consistently high levels of parathormone with a maximum value of $5.2 \mathrm{ng} / \mathrm{nl}$ were found in the brachiocephalic vein, suggesting a tumour in a lower parathyroid gland. At neck exploration ( $\mathrm{Mr} \mathrm{E}$ Milroy) an adenoma was found in the right lower gland and was removed. Postoperative serum $\mathrm{Ca}$ was normal.

Histological examination of the tumour from both mother and son showed a typical parathyroid chief-cell adenoma.

\section{Discussion}

The boy is interesting in two respects. He had primary hyperparathyroidism and a peptic ulcer which in children is a rare combination: Bjernulf et al. ${ }^{1}$ mentioned only 2 similar cases. In addition, it is unusual for a child with hyperparathyroidism to have no bone or renal involvement. ${ }^{3}$

The mother originally denied any symptoms, but early diagnosis before bony or renal complications had occurred was made when the serum $\mathrm{Ca}$ was measured in the child's close family. We consider that this should be done routinely in childhood hyperparathyroidism.

In neither patient was there any evidence of associated endocrine disturbance. This appears to be a further example of familial isolated hyperparathyroidism, which is rare and is said to be inherited as an autosomal dominant trait. ${ }^{2}$

In familial hyperparathyroidism generalised hyperplasia of the parathyroid glands is usually found. ${ }^{4}$ It is interesting that both our patients had a single parathyroid adenoma, which is the usual finding in childhood. ${ }^{5}$

We thank Dr J O'Riordan and Mr E Milroy for permission to publish details of these patients.

\section{References}

1 Bjernulf A, Hall K, Sjogren I, Werner I. Primary hyperparathyroidism in children. Brief review of the literature and a case report. Acta Paediatr Scand 1970; 59: 249-58.

2 Jackson C E. Hereditary hyperparathyroidism associated with recurrent pancreatitis. Ann Intern Med 1958; 49: 829-36.

3 Frier B M, Marrian V J. Uncomplicated hyperparathyroidism. Arch Dis Child 1974; 49: 808-10.

4 Cutler R E, Reiss E, Ackerman L V. Familial hyperparathyroidism. A kindred involving eleven cases, with a discussion of primary chief cell hyperplasia. $\mathrm{N} \mathrm{EnglJ} \mathrm{Med}$ 1964; 270: 859-65.

5 Verger P, Courand L, Guillard J M, Sandler B, Asanea K, Gambier P. Hyperparathyroidie majeure par adénome parathyroidien chez l'enfant. Ann Pediatr 1970; 17: 17-23.

Correspondence to $\mathrm{Dr}$ M W Moncrieff, Department of Paediatrics, The John Radcliffe Hospital, Oxford OX2 6HE.

\title{
Course of rotavirus gastroenteritis in a closed community
}

\author{
TSUNEO MORISHIMA, HIDEAKI YAMAGUCHI, SHOICHI NAGAYOSHI, \\ TAKAO OZAKI, SHIN ISOMURA, AND SAKAE SUZUKI
}

Department of Paediatrics, Nagoya University School of Medicine, Japan

SUMMARY There were 3 outbreaks of rotavirus gastroenteritis accompanied by milky-white stools in a closed community of 21 children in Japan during a 2-year period. Several different clinical courses were observed as a result of clinical, serological, and virological observations.

There is strong evidence from many parts of the world that human rotavirus is commonly associated with acute nonbacterial gastroenteritis in infants and children. ${ }^{1}$ In Japan, this virus is also found in acute gastroenteritis, particularly in that form of infantile diarrhoea which is accompanied by a milky-white stool (called Hakuri in Japanese). ${ }^{2}$ Hakuri has long been known as a well-defined and common type of nonbacterial gastroenteritis occurring in winter. ${ }^{3}$ Rotavirus has been detected by electron microscopy (EM) in the faeces from as many as $90 \%$ of Hakuri patients. ${ }^{24}$ However, the mode of human rotavirus infection and immunity against this virus has yet to be elucidated. To clarify these points, we studied a series of outbreaks of Hakuri in a closed community during a 2-year period.

\section{Materials and methods}

The study group comprised 21 children (10 boys, 11 girls) between ages 3 and 18 months, under the care of the Shuzenkai Orphanage in Nagoya. They 
lived together, mainly in the same room. There were 3 outbreaks of Hakuri between April 1976 and March 1978. The first two outbreaks took place in February and December 1977, and the third was in February 1978. As the affected children could not be isolated, the others were directly exposed. Throughout this period, using bedside charts, one of us looked daily for evidence of gastroenteritis, and recorded the following for each child: the presence of diarrhoea (a loose stool passed at least 3 times a day), fever $\left(37 \cdot 5^{\circ} \mathrm{C}\right.$ or more), vomiting, the duration of symptoms.

\section{Methods}

During each of the 3 epidemics, faecal samples were collected from between 3 and 5 Hakuri patients (a total of 11 patients, 12 samples) and from 7 asymptomatic children. These samples were examined by negative-staining EM. ${ }^{5}$ Immune adherence haemagglutination (IAHA) was also used to detect rotavirus antigen in faeces. ${ }^{6}$ Sera were examined by complement fixation (CF) using human rotavirus as an antigen source.

\section{Results}

During the 3 Hakuri outbreaks, 15 children had acute gastroenteritis clinically (Figure, black circles). Six were affected in the first outbreak, 3 in the second, and 6 in the third. Six children remained free of gastroenteritis symptoms. Ages of affected children ranged from 7 to 24 months (mean 13). Vomiting took place in all but one, particularly on the first day of illness. Loose milky-white stools were passed for one or two days and thereafter were greenish yellow. The incubation period appeared to be 24-48 hours. All 15 children recovered without complications. Except for the milky-white stools, these symptoms resemble those previously reported in winter epidemics of infantile gastroenteritis. The mechanism producing milky-white stools is still unclear.

At each outbreak, rotavirus was detected by EM or IAHA in all faecal samples collected from symptomatic children. Samples from 7 asymptomatic children were all rotavirus-negative. No other virus particles could be detected by EM.

Serological studies. The Figure shows that all 15 children with Hakuri developed CF responses to human rotavirus. CF antibody rise was absent in 3 of the 6 asymptomatic children during this period (Cases 7, 20, and 21), but was present in the remaining 3 (Cases 6, 18, and 19). Reinfection was observed in 2 patients. One (Case 11, 14 months), whose CF antibody titre was 4, had Hakuri in the second epidemic with a rise in titre to 16 . In another (Case

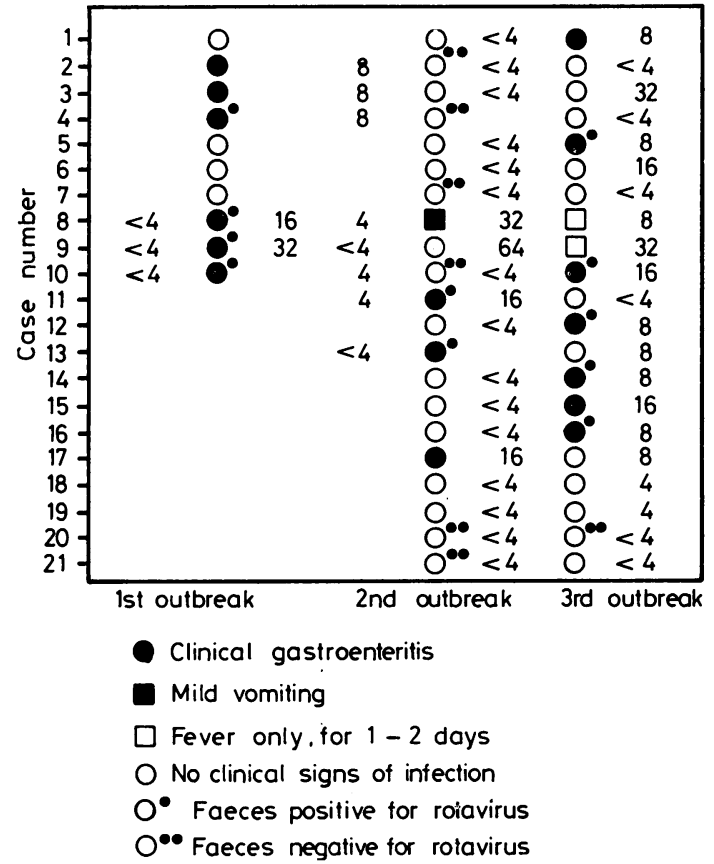

Figure Symptoms, and titres of $C F$ antibody to rotavirus, in 21 children during 2-year period.

10), the illness recurred after an interval of 12 months, with also an antibody rise from a titre of 4 to 16 . Two other children (Cases 8 and 9), who had suffered from Hakuri at the first outbreak, had mild vomiting or fever at one of the subsequent outbreaks (Figure, black squares and white squares), with a large renewed rise in CF antibody during the second outbreak.

\section{Discussion}

During a period of 2 years, five different clinical courses of rotavirus gastroenteritis were observed. Type 1, children who remained free of rotavirusinfection throughout all 3 Hakuri outbreaks (Cases 7, 20, and 21). Type 2, inapparent infection (Cases 6,18 , and 19), as reported among neonates by Murphy et al. ${ }^{7}$ Type 3 (the most common type), 13 children who developed Hakuri once but showed no clinical manifestations on re-exposure. Type 4, reinfection, was observed in 2 children (Cases 10 and 11) by EM of faeces and serological examinations. Type 5, exemplified by 2 children (Cases 8 and 10) who had typical Hakuri once, and later showed CF responses on re-exposure together with mild vomiting or fever. We can suppose that these children were reinfected with rotavirus, but that 
intestinal local immunity may have provided enough protection to prevent diarrhoea.

It has been suggested that there are subtypes of this virus, which might explain the variety of clinical courses. $^{8}$ We ourselves have suggested that immunological responses of this illness might differ from those in most other common viral infections, with individual differences in the acquisition of local immunity within the intestine. ${ }^{9}$

\footnotetext{
References

1 Walker-Smith J A. Rotavirus gastroenteritis. Arch Dis Child 1978; 53: 355-62.

2 Konno T, Suzuki H, Ishida N. Letter: Reovirus-like agent in Japanese infants with gastroenteritis. Lancet 1975; 1 : 918-9.

3 Sakamoto A, Iijima S, Suzuki S, Shirasawa S, Mori Y, Niimi M. Clinical and pathological studies on Hakuri, infantile diarrhoea with milky white stool. Ann Paediatrici 1964; 202: 293-302.
}

4 Morishima T, Ichikawa T, Yamaguchi H, et al. Acute infantile gastroenteritis caused by rotavirus in Japan. Eur J Pediatr 1978; 129: 259-65.

5 Bishop R F, Davidson G P, Holmes I H, Ruck B J. Detection of a new virus by electron microscopy of faecal extracts from children with acute gastroenteritis. Lancet $1974 ; 1$ : 149-50.

- Matsuno M, Nagayoshi S. Quantitative estimation of infantile gastroenteritis virus antigens in stools by immune adherence hemagglutination test. J Clin Microbiol 1978; 7: 310-1.

7 Murphy A M, Albrey M B, Crewe E B. Rotavirus infections of neonates. Lancet 1977; 2 : 1149-50.

8 Fonteyne J, Zissis G, Lambert J P. Letter: Recurrent rotavirus gastroenteritis. Lancet 1978; 1 : 983.

9 Morishima T, Nagayoshi S, Ozaki T, Isomura S, Suzuki S. Letter: Immunofluorescence of human reovirus-like agent of infantile diarrhoea. Lancet 1976; 2: 695-6.

Correspondence to $\operatorname{Dr} T$ Morishima, Department of Paediatrics, Nagoya University School of Medicine, 65 Tsuruma-Cho, Showa-Ku, Nagoya, Japan.

\title{
Primary vesicoureteric reflux in neonates with imperforate anus
}

\section{A M K RICKWOOD AND L SPITZ}

\author{
The Children's Hospital, Western Bank, Sheffield
}

SUMMARY Cystography, performed in 26 out of a total of 33 consecutive neonates with imperforate anus, revealed primary vesicoureteric reflux in 12 cases. The reflux was usually pronounced, and was particularly common in females and in infants with urinary tract infections. The incidence of reflux was unrelated to the severity of the anorectal anomaly, and reflux was often demonstrated in patients in whom an intravenous pyelogram had shown an undilated upper renal tract.

Primary vesicoureteric reflux (VUR) is the most common anomaly of the upper renal tract in childhood $^{1}$ and it was expected that this condition might often be found in infants with an imperforate anus, in whom the incidence of upper renal tract anomalies is such that a neonatal intravenous pyelogram (IVP) is generally considered mandatory. In four major series in which the genitourinary tract anomalies associated with imperforate anus were described, Singh et al. ${ }^{2}$ and Hasse $^{3}$ mentioned VUR but gave no statistics, while Wiener and Kiesewetter ${ }^{4}$ listed only 11 examples in a series of 200 cases. Smith ${ }^{5}$ was the only one to advocate a micturating cystourethrogram (MCU) as a routine in neonates with imperforate anus; he found 13 examples of VUR in
195 cases. At this hospital it was not formerly the practice to perform an MCU routinely in patients with imperforate anus, but when we did perform one in response to a particular indication (unexplained urinary tract infection, upper tract dilatation on IVP, to demonstrate a rectourethral fistula, etc.), VUR was often revealed, and reimplantation of refluxing ureters was virtually the only urological surgery undertaken in these patients. As a result, it is now our practice to perform an MCU in any new case of imperforate anus.

\section{Case material and findings}

33 new patients with imperforate anus presented as neonates in the 3-year period January 1976 to December 1978. Three died before their renal tracts had been investigated, and 4 had IVPs only. The remaining 26 had both IVPs and MCUs before being discharged from hospital. The radiological findings in these cases are shown in Table 1, and the comparison between the MCU and clinical findings in Table 2. Generally VUR of grades I and II only took place during voiding, and grade $\mathrm{III}$ during both filling and emptying of the bladder. Only one patient had a sacral deficiency likely to produce neurogenic bladder dysfunction; his MCU showed no reflux. It is too 